\title{
Towards G2G: Systems of Technology Database Systems
}

\author{
David Maluf and David Bell \\ NASA Ames Research center \\ MS 269/3 \\ Moffett Field, CA 94035 \\ 650-604-5000 \\ David.A.Maluf@nasa.gov
}

\begin{abstract}
We present an approach and methodology for developing Government-to-Government (G2G) Systems of Technology Database Systems. G2G will deliver technologies for distributed and remote integration of technology data for internal use in analysis and planning as well as for external communications. G2G enables NASA managers, engineers, operational teams and information systems to "compose" technology roadmaps and plans by selecting, combining, extending, specializing and modifying components of technology database systems. G2G will interoperate information and knowledge that is distributed across organizational entities involved that is ideal for NASA future Exploration Enterprise ${ }^{123}$. Key contributions of the G2G system will include the creation of an integrated approach to sustain effective management of technology investments that supports the ability of various technology. database systems to be independently managed. The integration technology will comply with emerging open standards. Applications can thus be customized for local needs while enabling an integrated management of technology approach that serves the global needs of NASA. The G2G capabilities will use NASA's breakthrough in database "composition" and integration technology, will use and advance emerging open standards, and will use commercial information technologies to enable effective System of Technology Database systems.
\end{abstract}

\section{TABLE OF CONTENTS}

1. INTRODUCTION............................................................ 1

2. OVERVIEW ......................................................... 2

3. APPROACH AND METHODOLOGY .......................... 3

4. APPLICATION INTEGRATION EXAMPLE ............... 4

5. CONCLUSIONS ........................................................... 6

REFERENCES ................................................................. 7

BIOGRAPHY ........................................................................ 7

${ }^{1}$ 0-7803-8870-4/05/\$20.00@ 2005 IEEE

2 IEEEAC paper \#1117, Version 4, Updated Jan 23, 2005

${ }^{3}$ http://exploration.nasa.gov/

\section{INTRODUCTION}

Technotogy is a central part of the U.S. National-Vision. Atlof the Strategic Technical Challenges for Sustainability identified in the NASA Exploration Systems Formulation Plan are related to technology, as are all of the identified systems challenges such as reusability, modularity, and autonomy. Technology is represented in technology databases.

Technology databases that support the management of technology investments are critical to the sustained success of the national vision. Technology databases has been identified as a key component in the acquisition processes (technology selection); and support of an integrated approach to the management of technoiogy has been identified as the main challenge. Such support will be framed by the overall spiral development process and strategy-to-task-to-technology approach.

The process of integrating technology management databases incorporates long-standing practices, such as the use of technology readiness levels (TRLS), and extending them with more recently developed space technology management tools and techniques. NASA has been pursuing linkage of database-independent technology management approaches to technology assessment and analysis, and risk management methodologies and tools.

Current approaches to integrating technology management data attempt to centralize important data and standardize important processes in order to address the issues of access and integration. Fundamentally, centralization is a response by organizations to their inability to deal comprehensively with distributed and disparate data, information, and knowledge that is stored or created with multiple computers using multiple applications with various file formats and data structures. This is also a response to their inability to provide integrated support for distributed and disparate processes that are tuned to each organization's and each individual's local needs.

When there is a lack of distributed and adaptive management and engineering information infrastructure 
architectures, there are incompatible databases, information services and management applications. These "islands" of incompatibility exist not only between organizations but also within them, where departments are unable to efficiently share key information. This same inability to deal with the distributed nature of work and the extreme fragmentation of data and information has prevented NASA from developing an integrated approach to the management of technology in the past, and prevents NASA from doing so now.

Centralization of technology databases and standardization of technology management processes across the diverse set of participants in NASA is infeasible and undesirable. While most research and development organizations use standard phase-gated processes to manage technology (E.g., [1-4]), studies show that enforcing a single management process across diverse research and development activities can significantly inhibit sustained innovation and effectiveness $([5,6])$.

One underlying aspect of the System of Technology Database Systems is to demonstrate the hypothesis that sustained effectiveness in the management of technology investment requires supporting a diversity of technology databases and applications customized for local needs rather than a one-size-fits-all or one-vendor approach. A second aspect to be delivered in the longer term is the hypothesis that automatic composition of technology analyses from multiple technology databases (including simulation models) is necessary for sustained effectiveness. This alternative frames the role for NASA leadership in advancing the science of information integration for systems of technology database systems.

\section{Objectives, including Specific Aims}

The general objective of $\mathrm{G} 2 \mathrm{G}$ is to provide an integrated approach to the management of technology investment for the Exploration Systems. Specific objectives include:

- The capability to enable decision making and system of technology database systems queries by finding, integrating, and composing models and data from technology databases, and running risk and lifecycle analyses and simulations

- A technology database capability for NASA to answer, based on requirements key measures and strategic technical challenges and on a priority basis, the needed capability and/or technology queries that may exist for short and long term goals.

- A proof-of-concept capability integrating multiple technology databases in support of spiral technology development at project, enterprise, agency and government levels.
- Enable sustainability over time to address the multigenerational aspect of the Exploration Enterprise (including inevitable changes in NASA personnel and changes in external contracts).

- $\mathrm{G} 2 \mathrm{G}$ is planned to impact systems development and testing within NASA as R\&D progresses and within the NASA research investment. In the context of the NASA Exploration Systems Formulation Plan, the G2G maps to the NASA Strategic Plan 2005 for all outcomes.

\section{OVERVIEW}

The function of the systems of technology database system is twofold: 1) integration - to develop integrated technology database testbeds for NASA mission directorates, 2) composition - to use the testbeds as a proof-of-concept for information synthesis in a system of technology database systems, namely a "composition" approach, leading to demonstration of the automatic composition of technology roadmaps and plans from program and project databases, simulation models and operational processes of heterogeneous fidelity for technology reporting, coordination, analysis, and decision-making.

In collaboration with selected NASA programs, G2G will baseline current NASA technology databases and technology management methods, develop a reference multi-vendor architecture using the IEEE standard for architectural descriptions of software-intensive systems [7], develop a multi-institution multi-database testbed using the architecture, and use spiral development to validate and improve the architecture relative to the baseline that changes with each spiral.

The integration approach for a System of Technology Database Systems supports the ability for various technology database system developers to customize databases and applications for local needs while enabling an integrated management of technology data that serves the global needs of NASA. NASA has defined a new database interface standard in partnership with industry to preserve government assets and avoid data and component migration and/or rewrites. Multi-technology vendors can thus use the same data over time.

Below are selected functions:

- The System of technology database systems approach in a context of use for composition (e.g., project- and enterprise-level technology); demonstrate the capability for a prioritized set of technology management applications answering queries on NASA key measures and strategic technical challenges (databases to be integrated include NASA Technology Inventory, Active Risk Management, Document Management). 
- System of technology database systems in multiple contexts of use including connection to NASA's Integrated Financial Management Program (IFMP).

The $\mathrm{G} 2 \mathrm{G}$ is a database product and requires strong competences in technology database and management of technology research, in enterprise quality storage and collaborative systems development, and in project management. G2G functions builds upon NASA successful collaborations with industry leaders in information technology. Key customers will be project, enterprise, agency and government stakeholders.

\section{APPROACH AND METhodology}

The overall approach to developing $G 2 \mathrm{G}$ is a system of systems approach which breaks down into two capabilities: a) composition - information synthesis for technology management decision making, and b) integrationintegrated access across multiple technology database systems.

\section{COMPOSITION}

The approach we take in the concept of operation of a System of Technology Database Systems is designed to retrieve answers to the concept of sustainability: Queries are framed to the notion of affordability, reliability/safety and effectiveness as the inherent parameters at the system and sub-system levels. System of Technology Database Systems can answer queries on system costs for design, development, test and engineering (DDT\&E). In other words, the overarching objective (hypothesis) of a System of Technology Database Systems is to answer- in queryable form - questions about the program and project investment portfolio and other NASA investments, composing management documents using multiple technology databases (Figure 1).

The fundamental approach to the problem of composition for the proposed system of technology database system is to construct a meta-database schema that maps parametrically (e.g. taxonomy decomposition and mapping) to strategic planning systems challenges (e.g. margins and redundancy, reusability, modularity, autonomy, data-rich virtual presence) to the technology investment database systems

\section{INTEGRATION}

The integration component challenge for structured and semi-structured information with diverse fidelity and pedigree is easier than the composition task. This component will be developed based on emerging open standards (e.g. World-Wide-Web Consortium (W3C) and Internet Engineering Task Force (IETF) standards) including XML[8] and XML extensions for content and representation (RDF, RSS)[9], XSLT[10], which is the standard for transformation of XML documents from one syntax to another, and WebDAV[11], another standard capability that defines HTTP extensions to sustain a distributed management of system of technology database systems. $\mathrm{XDB}[12]$, is a proposed extensible database standard that benefits from NASA patent-pending inventions that enable a schema-less approach for integration. NASA has defined the XDB standard in partnership with industry to preserve government assets and avoid data and component migration and/or rewrites for information sustainability over time $[13,14]$. Multitechnology vendors can thus use the same data and structures (Figure 2).

Current and future technology database systems have strong notions of interfaces. These interfaces allow binding to services provided by their corresponding architectures where their interfaces are described. XML is now accepted as the declarative interface and will be embraced as the core driver for a System of Technology Database Systems to allow database systems type definitions as the grammars and structures of the markup language.

\section{METHODOLOGY}

The architectural description (AD) for this approach is structured following the IEEE Recommended Practice for Architectural Description of Software-Intensive Systems (IEEE Std 1471-2000 [7]) iterative architecture for an evolutionary system. G2G builds upon a multi-institution testbed that integrates a variety of technology databases and management applications to serve as a proof-of-concept for the reference architecture. $G 2 \mathrm{G}$ provides integration across multiple information sources and the initial interoperation demonstration provides integrated access to mission technology databases (at the Johnson Space Center - JSC) and to NASA engineering databases through the Engineering Data Management System (EDMS). In a later phase, systems like IFMP, which is an agency wide MIS system for NASA business systems and processes, will be integrated into a Systems of Technology Database. We will demonstrate a Systems composition approach, leading to a sequence of demonstrations of automatic and project databases simulation models and operational processes of heterogeneous fidelity for technology reporting, coordination, analysis, and decision-making. The table below describes the databases and applications that $G 2 G$ is integrating in the initial testbeds. The XDB Application Programming Interfaces (API) enables systems support for human-computer, computer-computer, and human-human communications. The "Program Management ToolErasmus Application Integration" is one example of how the emerging NASA standard has been used to integrate technology databases for the management of technology. This applications is described in detail in the following section. Figure 1 illustrates how the API can be used to compose custom reports from a diversity of technology 


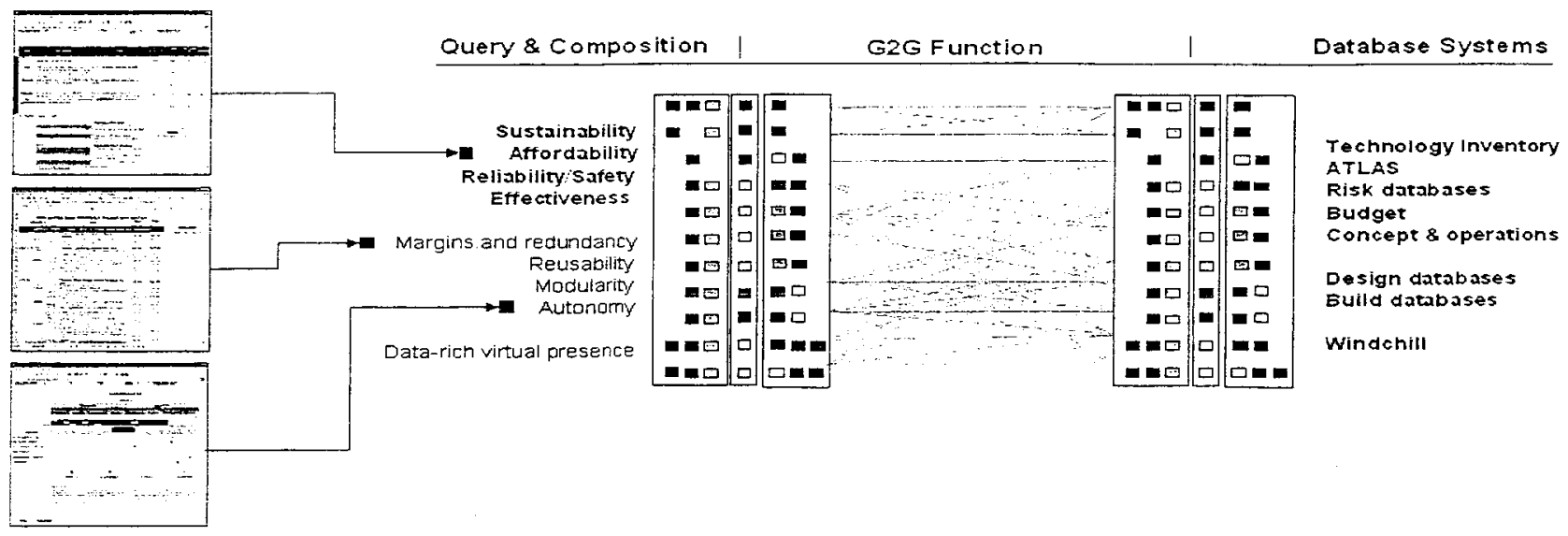

Figure 1. Illustration of the composition of a project plan from a diversity of technology databases and component databases.

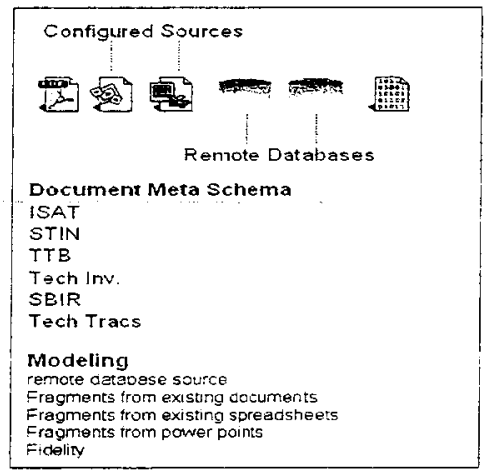
delity
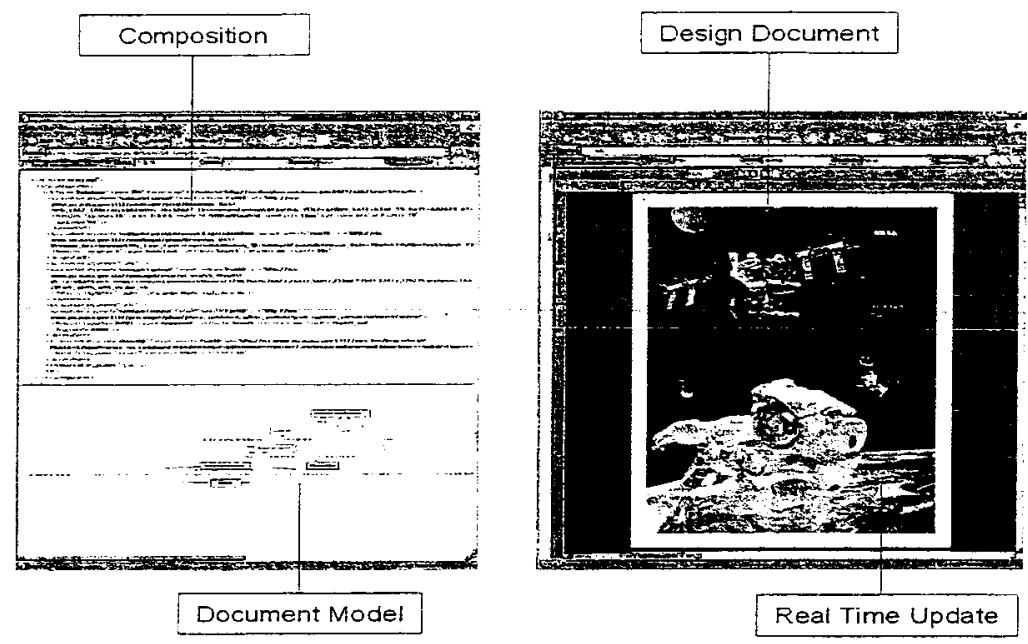

Figure 2. Diversity of technology management applications for tasks such as technology maturation and gap analysis to utilize a diversity of technology databases.

databases. G2G works to coordinate effectively with and, where possible, leverage other technology database investments. As a subsystem, $G 2 G$ institutes a selfsupporting H\&RT system component for Project Prometheus and the Centennial Challenges program. External to exploration systems goals, G2G is a capability that can interoperate with other enterprises concerning $R \& D$ and with other agencies.

\section{APPLICATION INTEGRATION EXAMPLE}

We present a description of an actual application integration example where interoperation is provided across two major NASA agency wide applications.

The Erasmus Program Management System is a NASA agency-wide program management system. Erasmus is a dashboard that provides Project Managers, Program Managers, Theme Directors, and Associate Administrators with an information systems tool that supports the management accountability and performance measurement process. Projects and programs can be sorted and viewed by Enterprise, Project Type, Theme, Stoplight Status, or many other parameters. The Erasmus system is updated monthly to include key accomplishments, top issues, reserves status, risk matrix, estimate to complete, quality and performance indicators, and human capital assessment schedule status.

The NASA ECS (Engineering for Complex Systems) Program uses a customized system, PMT (Program Management Tool), which allows insight into program management at a finer detail. Thus, to support both Program and Agency requirements, ECS is required to periodically synchronize the information between systems. 
Table 1. Databases and applications integrated

\section{Description}

NTI NASA Technology Inventory - Enterprise-level tracking

ARM Active Risk Manager - Managing project-level technology risks

Windchill Product data management at JSC, OExS and other NASA centers

IFMP NASA HQ agency-level Integrated Financial Management

RAD / JPL NASA risk and anomaly database; mishap anomaly database

$-\mathrm{ISAB}$

-Inter-center-System Analysis Data Base-

The ultimate goal is to provide a seamless integration between PMT with Erasmus, to improve ECS productivity and provide timely and accurate information to Agency and Program managers by eliminating the need to update both management systems and improve data consistency between them.

Interoperability can be said to mean that the functional characteristics of a system should stay constant across different operating system platforms, programming languages, data models and hardware. This problem is difficult to solve when the systems are based on differing architectures. Erasmus is based on Macromedia ColdFusion, and PMT is based on J2EE (Java 2 Enterprise Edition), each having a preferred communication protocol. Several standards are used to address this architectural mismatch including: 1) Extensible Markup Language (XML) as the interlingua, 2) the emerging NASA XDB extensible database standard for query-based XML transformation and 3) the W3C Simple Object Access Protocol (SOAP). Web Services enabled by these standards offer a greatly simplified method of client/server and server/server communications. Web Services enabled by these standards offer a greatly simplified method of client/server and server/server communications. Previously, low-level programming methods were required to implement this distributed processing model. With Web Services, communication and transactions are facilitated using the universally understood XML (Extensible Markup Language) over HTTP, the same method of communication for web browsers. This approach also solves problems with communication through firewalls. To integrate these architecturally different systems, a Web services interface to
Erasmus was created and an Erasmus XML template document was defined.

In this Web services interface, Erasmus business methods were exposed to clients such as PMT for direct consumption. Web services are services offered by an application to other applications via the Internet. Web services depend on the ability of parties to communicate with each other even if they are using different information systems. A Web service, a server application that implements the procedures that are available for clients to call, is deployed in a server-side container, describes itself using Web Services Description Language (WSDL[16]) and is transported via SOAP over the World Wide Web standard transport protocol, HTTP or HTTPS. Erasmus exposes its business methods to potential clients by describing itself using a WSDL description document. A WSDL description is an XML document giving all the relevant information about the Web service such as the operations that can be called on it and the parameters for those operations.

SOAP defines a framework for the exchange of XML documents. It specifies, among other things, what is required and optional in a SOAP message and how data can be encoded and transmitted. Since security is a major concern for all parties involved, and given that the payload travels across the internet, authentication, authorization and encryption needed to be addressed. This was achieved by sending a SOAP message across the wire encrypted using Secure Sockets Layer (SSL) over HTTP, commonly referred to as HTTPS. Authentication and authorization was handled by Erasmus checking for a valid URI (e.g.: authorization) with a matching UUID (e.g.: authentication). 

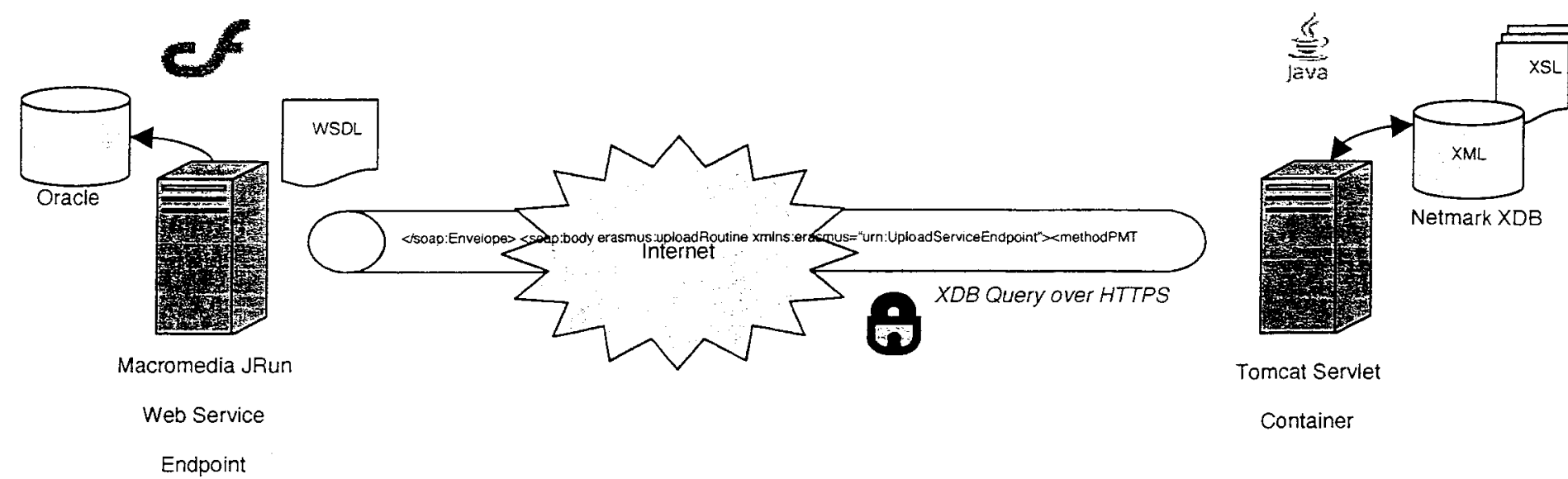

Container

Erasmus Reporting

Program

Tool

Management Tool

(PMT)

Figure 3. Erasmus-PMT Integration: System Architecture

In addition to creating a service endpoint for clients, Erasmus also defined the standard Erasmus XML template document containing the Erasmus specific business terms that will be completed by each client and sent within the SOAP payload. Any and all clients who wish to integrate with Erasmus must comply and map their data to the Erasmus template document schema. In order for PMT to comply, a data transformation was required as a preprocessing step before upload.

Integration Steps:

- Erasmus creates a standard Erasmus XML template document defining the Erasmus data model Erasmus creates a WSDL description and business method implementations, and then deploys them as Web services.

- Using the WSDL description, PMT creates Java client stubs, low-level classes that are needed by a client to communicate with a remote web service.

Runtime Scenario:

1. An ECS user logs into PMT where the monthly report is entered and submitted.

2. PMT makes the necessary calls to Netmark and applies Extensible Style Sheets (XSL) to transform the XML data to conform to the standard Erasmus XML template document.
3. PMT instantiates an Erasmus client stub and calls the appropriate business method, passing the XML data as a method parameter.

4. Erasmus begins processing the request by authenticating and authorizing the transaction, then parsing the XML document and updating the Erasmus backend.

5. Erasmus sends a return code back to PMT..

What is the returned to the user is an integrated document report in a format that is customizable by the end user. Information from various documents in extracted and integrated to form the final integrated document (report).

\section{ConClusions}

$\mathrm{G} 2 \mathrm{G}$ is enabling locally and globally optimized management of technology in NASA through two main mechanisms: 1) integration, and 2) composition. This improves communication across multiple stakeholders including review board, project and line management, and project team members. Improved communications around technology is critical to NASA's future. 


\section{REFERENCES}

1. J.R.Wertz and W.J.Larson, Space Mission Analysis and Design. 1999, Boston: Kluwer.

2. NASA Procedural Requirements NPR $7120.5 B$ (November 21, 2002), NASA Program and Project Management Processes and Requirements, NASA Office of the Chief Engineer (Code AE) at NASA Headquarters.

3. K.B.Clark and T.Fujitomo, Product Development Performance: Strategy, Organization, and Management in the World Auto Industry. Boston MA. 1991: Harvard Business School Press.

4. S. Wheelwright and K.B.Clark, Revolutionizing Product Development: Quantum Leaps in Speed, Efficiency and Quality. 1992, New York: The Free Press.

5. D.G.Bell, S.Newman, and N.Repenning. Process, Practice and Politics: Understanding the Relationship between Documentation, Depioyment, and Daily Work. in Academy of Management Conference: Organization and Management Theory Division. 2002. Denver, CO.

6. Christensen, C., The Innovator's Dilemma: When New Technologies Cause Great Firms to Faii. 1997: Harvard Business School Press.

7. IEEE Std 1471-2000 IEEE Recommended Practice for Architectural Description of Software-Intensive Systems.

8. XML. http://www.w3.org/XML

9. RDF. http://www.w3.org/RDF/.

10. XSLT. http://www.w3.org/TR/xslt.

11. Whitehead, J. and Y. Goland. WebDAV: A network protocol for remote collaborative authoring on the Web. in CSCW. 1999.

12. Maluf, D., P. Tran, and T. La. "An Extensible 'Schemaless' Database Framework for Managing High-

Throughput Semi-Structured Documents. in IASTED, Applied Informatics. 2003.

13. D.Maluf, Managing Semi-structure Data Dynamically. Foundations of Intelligent Systems, LNCS, 2003': p. 231-241.

14. D.Maluf and P.Tran, 'Articulation Management for Intelligent Integration of Information. Systems, Man, and Cybernetics, 2001. 31(4).

15. SOAP. http://www.w3.org/TR/soap/.

16. WSDL http://www.w3.org/TR/wsdl.

\section{BIOGRAPHY}

David A. Maluf leads the NASA Advanced Exploration

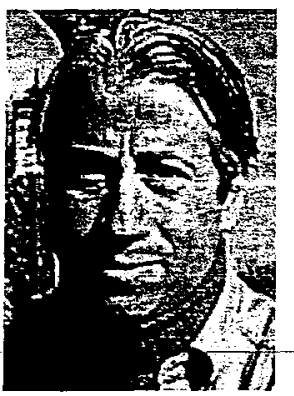
Network laboratory (AEN), a laboratory consisting of $20+$ staff with an average of 12 projects/year. He has over 70 technical publications in journals and conference proceedings, over hundreds of presentations at international conferences and is an inventor of numerous patents. He has taught courses on system engineering and databases, and has written two books. He received his $\mathrm{PhD}$ from McGill University and conducted post-doctoral research in information integration at Stanford University.

David G. Bell is the Director of the Research Institute for Advanced Computer Science, located at the NASA Ames Research Center. He received his $\mathrm{Ph.D}$. from Cornell University, worked for ten years at the Xerox Palo Alto Research Center, and previously held an appointment as visiting lecturer at MIT where he managed one of the four research programs in the MIT Center for Innovation in Product

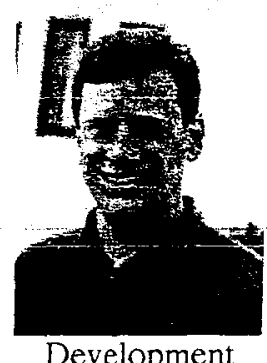

Development 